\section{Attitude pratique en cas d'un accident vasculaire cérébral: diagnostic}

\author{
Groupe de travail suisse sur l'accident vasculaire cérébral (GTSAVC) \\ et Fondation suisse des maladies cardiaques (FSMC) ${ }^{1}$
}

\begin{abstract}
Une thrombolyse rapidement mise en œuvre en cas d'une ischémie cérébrale améliore avec une haute probabilité le destin du patient. Chez 15\% des patients, la thrombolyse prévient un handicap physique permanent. Ainsi, il est nécessaire d'instruire le public et le personnel médical soignant concernant les signes et les symptômes de l'AVC afin de pouvoir agir rapidement. Dans la clinique, nous réalisons d'abord une évaluation neurologique, puis un examen neuroradiologique (TDM ou IRM). Si ces investigations mettent en évidence une ischémie nous procédons à une thrombolyse intraveineuse ou intra-artérielle, selon le temps écoulé et les possibilités du centre en question. Après, suivent une évaluation ultra-sonologique des vaisseaux cérébraux, des investigations cardiaques (ECG, échocardiographie) et des examens d'imagerie et de laboratoire additionnels, qui seront à la base d'une prophylaxie secondaire adaptée individuellement.
\end{abstract}

\footnotetext{
1 Les personnes suivantes ont participé par une contribution grande ou petite à la rédaction des articles sans avoir reçu la moindre indemnité financière:

M. Arnold, Berne; A. Barth, Berne; C. Bassetti, Berne; R. Baumgartner, Zurich; S. Beer, Valens; J. Bogousslavsky, Lausanne; A. Carota, Lausanne; A. Carruzzo, Lausanne; G. Devuyst, Lausanne; A. Dupont, Bâle; S. Engelter, Bâle; P. Erne, Lucerne; F. Fluri, Bâle; R. Guzman, Berne; S. Heffet, Genève; S. Heffet, Genève; HJ. Hungerbühler, Aarau; HG. Imhof, Zurich; E. Keller, Zurich; H. Keller, Zurich; J. Kesselring, Valens; J. Le Floch-Rohr, Genève; K. Loevblad, Berne; Ph. Lyrer, Bâle; F. Mahler, Berne; A. Marx, Lugano; H. Mattle, Berne (rédacteur de cet article); B. Meier, Berne; R. Mordasini, Berne; M. Mosso, Zurich; F. Müller, Scherzingen; S. Osswald, Bâle; M. Reichhart, Lausanne; L. Remonda, Berne; A. Rivoir, Berne; D. Rüfenacht, Genève; W. Rutishauser, Genève; R. Sztaijzl, Genève; L. Regli, Lausanne; J. Rem, Bâle; A. Rivoir, Berne; G. Schroth, Berne; C. Städler, Lugano; P. Stierli, Aarau; M. Sturzenegger, Berne; B. Tettenborn, St-Gall; C. Tosi, Lugano; B. Weder, St-Gall; S. Windecker, Berne.
}

Correspondance:

Prof. Heinrich Mattle

Neurologische Klinik und Poliklinik

Inselspital

CH-3010 Berne

Tél. 0316322111

Fax 0316329679

e-mail heinrich.mattle@insel.ch

\section{Objectif d'apprentissage}

1. Connaissance de base en Neuroradiologie (TDM, IRM)

2. Signification diagnostique de l'ultrason neurovasculaire

3. Méthode de diagnostic cardiaque et interprétation des rapports

\section{Marche à suivre pendant les premières heures}

La thrombolyse peut apporter à certains patients souffrant d'AVC une aide décisive pendant les premières heures. Il en résulte la nécessité d'informer le public sur les signes et les symptômes de l'AVC. En cas d'hospitalisation rapide dans un centre de référence pour les AVC on peut offrir de l'aide à la personne concernée et lui donner une chance d'améliorer son destin. En cas d'urgence, le médecin de famille, le médecin d'urgence ainsi que le personnel soignant devraient être en mesure de reconnaître un AVC et ainsi l'urgence. Si la suspicion d'un AVC se confirme après évaluation neurologique à la clinique, une imagerie cérébrale devrait être pratiquée d'urgence, soit une tomodensitométrie soit une résonance magnétique nucléaire, afin d'exclure une hémorragie ou d'autres pathologies (tableau 1). En cas d'ischémie, on procède sans délai à une thrombolyse intraveineuse ou à une artériographie cérébrale suivie d'une thrombolyse intra-artérielle en cas d'occlusion d'un vaisseau.

\section{Marche à suivre après les premières heures (et également après thrombolyse)}

Si les circonstances le permettent, le prochain pas devrait être un examen ultra-sonographique neurovasculaire des vaisseaux exo et intracrâniens. Si l'anamnèse, l'examen cardiaque clinique et TDM ou IRM évoquent en premier lieu une embolie cardiogène, on procédera à des examens complémentaires moyennant une échocardiographie trans-thoracique ou trans-œsophagienne [1]. Par rapport à l'approche trans-thoracique l'échocardiographie trans-oesophagienne est plus sensible en particulier concernant une pathologie atriale ou valvulaire ou pour des plaques de l'arc aortique. Pour cette raison, il est préférable de pratiquer en premier lieu une échographie transoesophagienne, surtout chez les patients plus jeunes. S'il y a une suspicion d'un trouble du rythme, un électrocardiogramme de longue durée (ECG-holter) devrait être pratiqué, au cas où une fibrillation auriculaire n'a pas déjà été démontrée à l'ECG de repos.

Si les causes de l'AVC demeurent obscures après ces premières investigations complémentaires, nous recommandons des examens de laboratoire complémentaires selon le tableau 2. 
Dans des situations peu claires, une IRM cérébrale est toujours indiquée [2, 3]. Des infarctus lacunaires, par exemple sont souvent visibles uniquement à l'IRM. Des hématomes pariétaux, des dissections artérielles sont uniquement démontrables à l'IRM et pour des thromboses des sinus ou veines intra-cérébrales, l'IRM est l'outil diagnostic de premier choix. Dans les situations d'étiologie claire, l'IRM peut donner des informations plus précises concernant la localisation et l'importance de la lésion ischémique, mais n'influencera guère les mesures prophylactiques futures.

\section{Tomodensitométrie (TDM) [4]}

Avec une TDM cérébrale, il est possible de distinguer de façon fiable entre une hémorragie intracrânienne et un AVC ischémique [5]. Dans les premières heures de l'AVC ischémique, la TDM peut être normale. Avec les tomographes modernes, à haute résolution, il est possible de visualiser le thrombus ou l'embole d'une occlusion vasculaire aiguë comme signal hyperdense

\section{Tableau 1}

Examen d'urgence en cas d'un déficit neurologique aigu focal.

Tomodensitométrie ou tomographie par résonance magnétique du cerveau (également angio-TDM ou angio-IRM)

Angiographie sélective (comme moyen diagnostic et pour thrombolyse)

Ultrasonographie neuromusculaire (au centre qui ne procède pas à des angiographies)

Electrocardiogramme, si nécessaire monitoring-ECG

Examen du sang: VS, CRP, hémoglobine, hématocrite, leucocytes et répartition, thrombocytes, sodium, potassium, taux de prothrombine, bilan lipidique, osmolarité du sérum*, examen urinaire*, radiographie du thorax*

Electroencéphalogramme (uniquement en cas de suspicion de crises d'épilepsie avec paralysie post-ictale)

Ponction lombaire (en cas de suspicion d'hémorragie sous-arachnoïdienne et TDM négatives ou suspicion d'un processus infectieux)*

Surveillance continue de la pression artérielle*

Oxymétrie*

* Examens complémentaires facultatifs

\section{Tableau 2}

Examen de laboratoire en cas de déficit neurologique focal aigu (phase 2).

Sérologie pour syphilis, Borrélia, VIH, varicelle-/zoster

Electrophorèse des protéines

Facteur antinucléaire, facteur rhumatoïde et d'autres auto-anticoprs

Electrophorèse de l'hémoglobine (chez des patients noirs ou provenant de régions méditerranéennes)

Anticorps anti-phospholipides

Protéine $\mathrm{C}$, protéine $\mathrm{S}$, anti-thrombine-3, facteur de résistance contre protéine $\mathrm{C}$ activée, dosage du facteur 5 (ces dosages devraient être effectués seulement quelques semaines après la phase)

Ponction lombaire

\section{Figure 1}

TDM d'un patient avec un hémisyndrome aigu gauche. Le thrombus dans l'artère cérébrale moyenne droite est visible sous forme d'un ruban hyperdense (hyperdense artery sign). Cependant les altérations du parenchyme ne sont pas encore visibles (Source: Unité de Neuroradiologie, Hôpital de l'île, Berne).

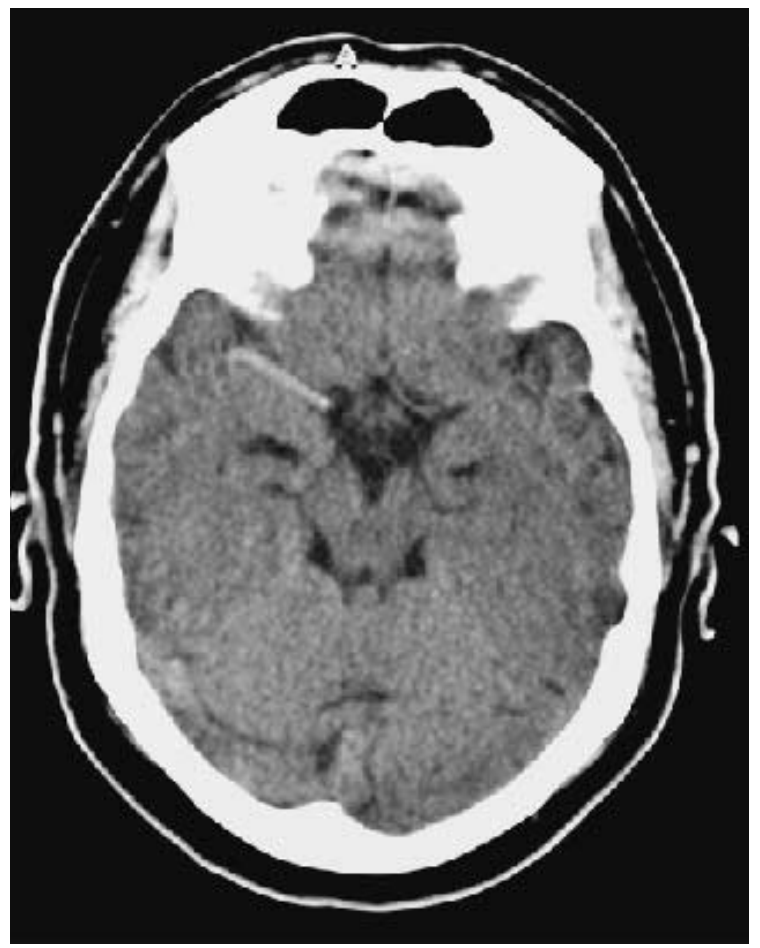

intra-artériel ou de reconnaître déjà à trois heures après l'occlusion vasculaire des signes directs ou indirects $d u$ changement parenchymateux ischémique (figure 1) [6]. L'ischémie a pour conséquence une diminution de l'absorption des rayons $\mathrm{X}$ de la substance grise. En cas d'une occlusion de l'artère cérébrale moyenne, les ganglions de la base et le cortex cérébral perdent en densité et sont moins bien délimités de la capsule interne et externe et de la substance blanche, et cela longtemps avant que l'hypodensité indique de façon indéniable l'infarcissement cérébral. Par la suite, un œdème du tissu infarci et une compression des espaces du liquide céphalo-rachidien externe et interne peuvent survenir. L'angio-TDM donne une visualisation directe des sténoses et occlusions vasculaires possibles. Elle se base sur la technique de la TDM-spiralée associée à une application de produits de contraste intraveineux et une reconstruction tri-dimensionnelle des vaisseaux cérébraux assistée par ordinateur. La génération la plus récente des TDM-multidétecteurs peut également être utilisée pour des mesures perfusion du parenchyme cérébral.

\section{Résonance magnétique nucléaire (RMN) (figure 2)}

La résonance magnétique spin-écho conventionnelle produit les images sur la base du contenu en eau des tissus. Ainsi l'infarcissement cérébral peut seulement 


\section{Figure 2}

Tomographie de résonance magnétique nucléaire d'un patient atteint depuis 3 heures d'une hémiparésie gauche à prédominance facio-brachiale (il s'agit du même patient qui est illustré dans la figure 5). Figures A à C: sont prises avant, figure D à F sont prises après thrombolyse de l'artère cérébrale moyenne. Figure A: Image de spin-écho pondérée en T2, qui est normale et qui ne peut pas mettre en évidence la zone mise en danger par l'ischémie. Figure B: Sur l'image diffusion, on reconnaît clairement la zone ischémique grâce à son hyper-signal. Figure C: Cette même région se montre comme zone de circulation sanguine défaillante à l'image de perfusion (image de «time to peak». L'image spin-écho pondérée en T2 montre une altération du signal très discret dans la région centrale droite après thrombolyse réussie (figure D), et l'anomalie du signal à l'image de diffusion s'est presque normalisée (figure E). On reconnaît l'anomalie de circulation sanguine dans la région concernée à l'image de perfusion (source: Unité de neuroradiologie, Hôpital de l'île, Berne).
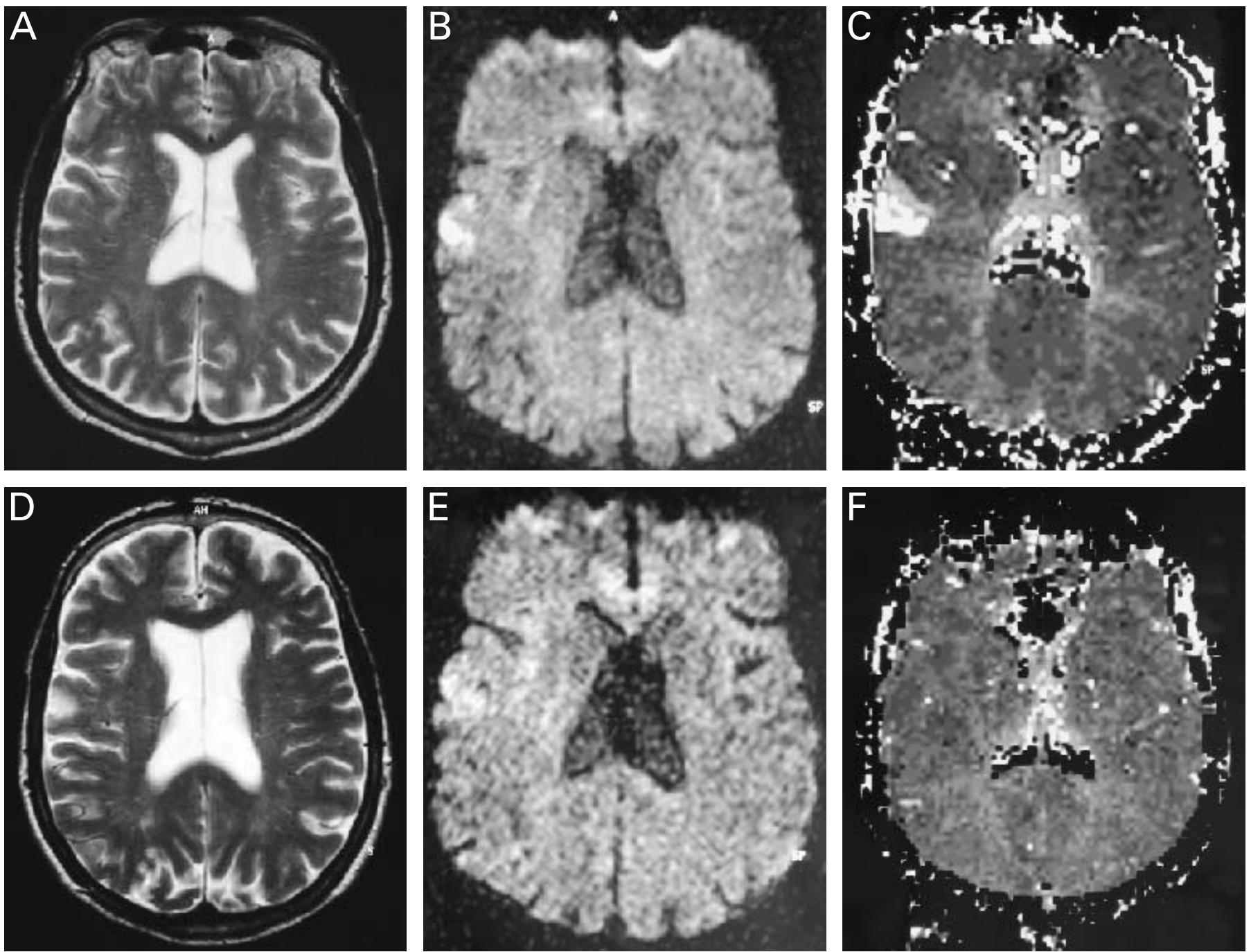

être visualisé au bout de quelques heures avec l'apparition d'un œdème cérébral cytotoxique et vasogène. La reconnaissance précoce de l'œdème cytotoxique est rendue possible depuis peu d'années à l'aide de l'imagerie de diffusion [7]. Les séquences qui génèrent l'image seront modifiées de façon à ce que les mouvements de l'eau du parenchyme peuvent être démontrés. En cas d'œdème cytotoxique, l'eau sera déplacée vers le compartiment intracellulaire et, ainsi la mobilité de la plus grande quantité d'eau sera restreinte, et la diffusion diminue. Ceci est mesuré à l'aide de la valeur CDA (coefficient de diffusion apparent). Une valeur CDA abaissée correspond à un signal augmenté à l'image de diffusion. Si un œdème vasogénique et l'infarcissement du tissu succèdent à l'œdème cytotoxique, les barrières pour les mouve- ments d'eau diminuent, la valeur CDA augmente et le signal à l'image de diffusion diminue après les premiers jours [8]. Le problème technique de l'imagerie de diffusion réside dans les artéfacts de mouvements, c'est pourquoi une application clinique large peut être attendue seulement avec la nouvelle génération des tomographies RMN et une production très rapide de l'image. Aussi, la tomographie RMN de perfusion va passer de la recherche expérimentale à la clinique. Elle se base sur la visualisation du changement de signal dans le parenchyme cérébral causé par le passage d'un bolus d'une substance de contraste à travers les vaisseaux cérébraux, en particulier des capillaires cérébraux. Habituellement, le signal chute lors du passage du bolus, mais pas dans la zone d'infarcissement. L'angiographie RMN donne la possibilité de démon- 
trer de façon non invasive les grands vaisseaux cérébraux intra et exo-crâniens et ainsi des sténoses et occlusions. En cas d'AVC, l'interruption d'un vaisseau peu indiquer de façon indirecte un infarcissement, même avant que le changement parenchymateux puisse être reconnu à l'image spin-écho. Des séquences dites pondérées sur la susceptibilité permettent de découvrir des hémorragies cérébrales et de les distinguer de l'ischémie déjà au stade hyper-aigu [9].

\section{Ultrason neurovasculaire (figure 3)}

Diverses méthodes ultrasonographiques sont utilisées pour les artères cérébrales exo et intracrâniennes $[10,11]$. Moyennant la sonographie Doppler exo et transcrânienne on peut mesurer la vitesse et la direction du flux sanguin. La sonographie duplex complète la mesure Doppler du flux sanguin par la démonstration du tissu dans l'image-B. Ainsi les échos reflétés par des structures fixes sont visualisés dans différents niveaux de la grille d'une coupe d'image bidimensionnelle selon l'intensité et la localisation de structure reflétée. Dans la sonographie duplex codée par couleur et Doppler-puissance le spectre de fréquence et l'intensité du signal Doppler sont visualisés en couleur. Si une visualisation du flux sanguin n'est pas possible par le Doppler exo où transcrânien, le Duplex-couleur, où en cas d'une résistance endocrânienne du flux, des produits de contraste peuvent améliorer le rendement diagnostic. La sonographie Doppler est propice à la détection de sténoses, occlusions, spasmes, des réseaux circulatoires collatéraux et des embolies dans les artères exo et intracrâniennes grâce au Duplex-couleur, à la sonographie duplex, les altérations de la paroi vasculaire comme les plaques athéromateuses ou la dissection peuvent être démon- trées. Concernant les plaques, nous nous intéressons surtout à la localisation et à la taille, l'échogénicité et la surface. Par contre, la sonographie n'est pas utile pour démontrer ou exclure la présence d'anévrisme intracrânien.

\section{Angiographie (figures 4 à 6)}

Pendant l'angiographie cérébrale, un cathéter est introduit dans l'artère fémorale, avancé sélectivement dans un vaisseau cérébral donné et injecté avec un produit de contraste afin de permettre la visualisation par rayons X. Dans la plupart des cas, l'angiographie cérébrale sert à la caractérisation ou à la vérification d'un processus vasculaire pathologique, comme il a été détecté à la TDM, l'IRM ou à l'examen ultra-sonologique neurovasculaire ou encore comme il a été suspecté sur la base de la clinique. Les indications les plus importantes englobent la preuve ou l'exclusion de sténoses, occlusions, fistules, anévrismes ou d'artériopathies spécifiques. L'angiographie est également à la base de la neuroradiologie interventionnelle, qui permet un traitement local de la pathologie vasculaire en cas d'un AVC aigu à l'aide de la thrombolyse intra-artérielle [12].

\section{Diagnostic cardiaque}

Des sources cardiaques emboligènes sont tenues responsable pour 20 à 30\% de tous les AVC ischémiques, chez les patients jeunes (moins que 45 ans) jusqu'à 40\% [13]. Le diagnostic clinique d'AVC cardio-embolique est indirect dans la plupart des cas et nécessite la preuve d'une source cardio-embolique possible et l'exclusion d'une étiologie cérébrale vasculaire (tableau 3) [14].

\section{Tableau 3}

Source cardiaque emboligène et la localisation au niveau du cœur.

\begin{tabular}{ll}
\hline Maladies & Localisation possible des sources emboligènes \\
\hline Infarctus du myocarde aigu & Ventricule akinétique, interaction de la surface de l'endocarde \\
\hline Infarctus du myocarde ancien associé à un anévrisme & $\begin{array}{l}\text { Au niveau de l'anévrisme surtout thrombus ventriculaire gauche } \\
\text { localisé au niveau de la pointe du cœur }\end{array}$ \\
\hline Cardiomyopathie, dilatée & Thrombi au niveau des oreillettes ou des ventricules \\
\hline Sténose mitrale d'origine rhumatismale & Thrombus au niveau de l'oreillette ou de l'auricule dilaté \\
\hline Endocardite infectieuse & Végétation à la surface des valves ou au niveau de l'appareil valvulaire \\
\hline Sclérose de l'anneau mitral & Thrombi au niveau de l'anneau valvulaire \\
\hline Myxome de l'oreillette & Plus souvent localisé au niveau de la fossa ovalis à gauche \\
\hline Fibrillation auriculaire & Thrombus au niveau de l'oreillette ou de l'auricule gauche \\
\hline Maladies de l'oreillette avec bradycardie ou tachyarythmie & Thrombus au niveau de l'oreillette ou de l'auricule gauche \\
\hline Foramen ovale perméable, anomalie de septum atrial & $\begin{array}{l}\text { Embolie paradoxale de thrombi veineux dans la circulation artérielle, } \\
\text { surtout en cas d'association avec un anévrisme du septum interatrial }\end{array}$
\end{tabular}




\section{Figure 3}

A Duplex-couleur de la dissection carotidienne d'un homme de 50 ans en bonne santé.

B Duplex-couleur d'une bifurcation carotidienne d'une femme de 70 ans après plusieurs AIT hémisphériques gauches. La visualisation de flux sanguin est morcelée sur cette image en couleur, en raison des ombres sonographiques induites par les altérations artério-sclérotiques des parois vasculaires (CCA = arteria carotis communis, ICA = arteria carotis interna, ECA = arteria carotis externa). Des accélérations du flux et des turbulences, que l'on reconnaît au pattern des couleurs perturbé, indiquent une sténose dans cette région.

C Visualisation isolée de la fin de l'artère carotide interne.

D Profil de flux issu de l'endroit le plus étroit de la sténose. Avec une vitesse de $480 \mathrm{~cm} / \mathrm{sec}$ de vitesse systolique maximale, le flux est très accéléré et indique de ce fait une sténose de 70\%. Comparé avec la figure 4. (source: Clinique de Neurologie, Hôpital de l'île, Berne).

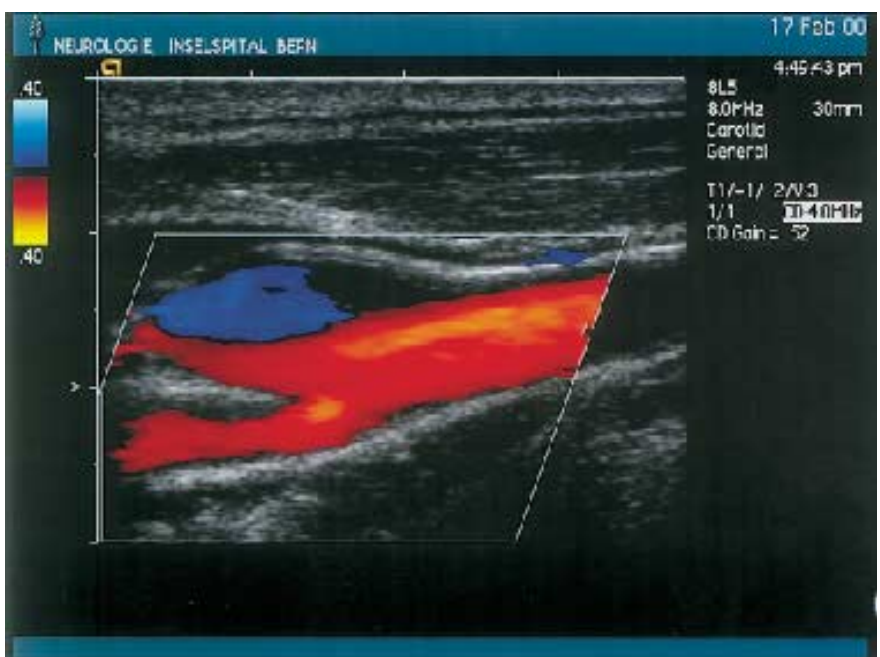

A

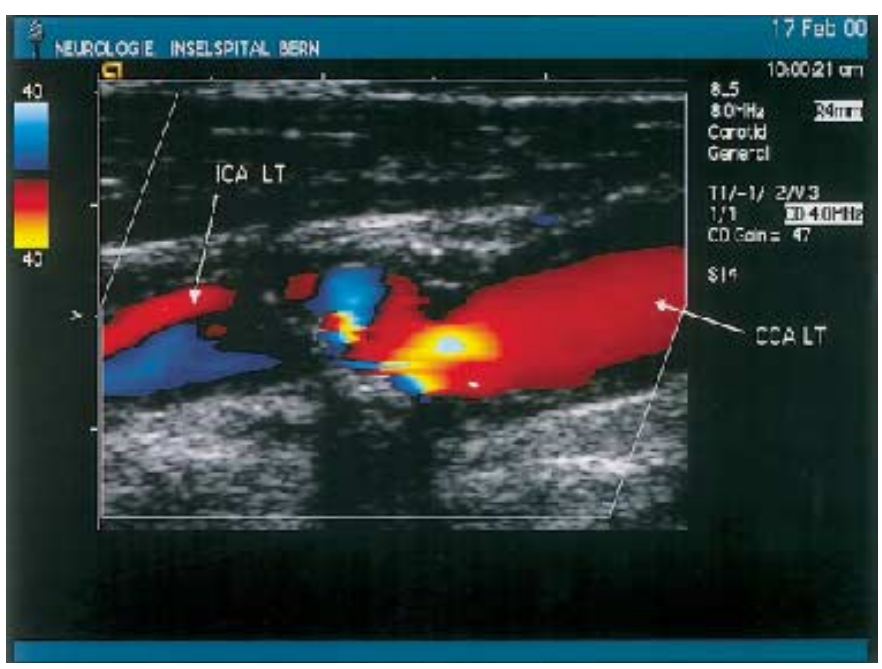

$\mathrm{C}$

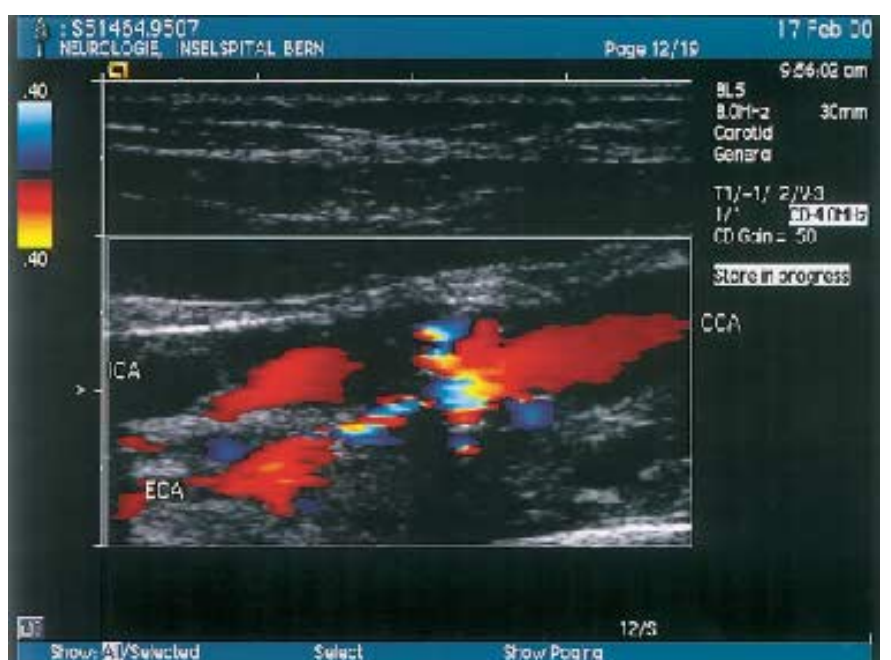

B

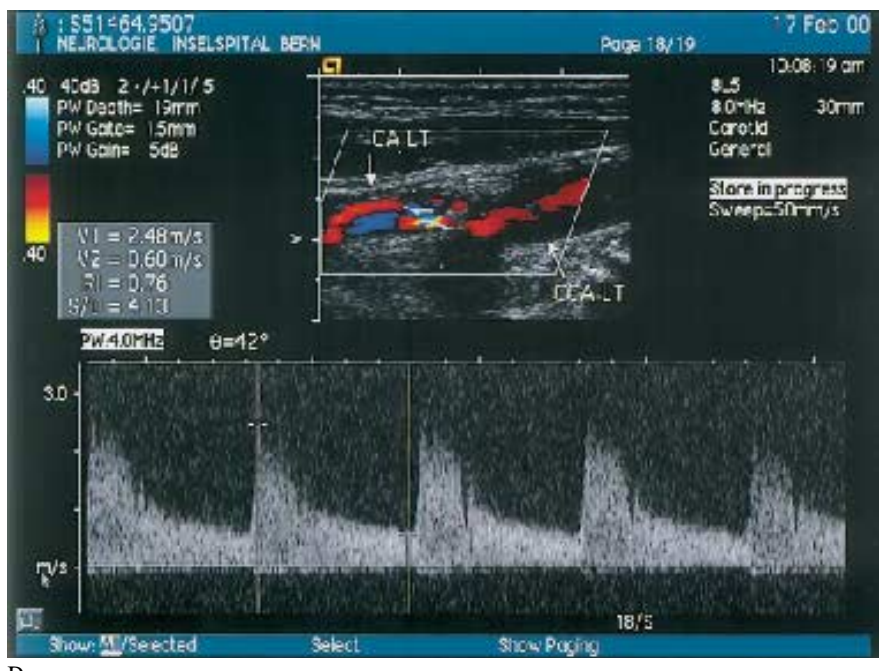

Electrocardiogramme (ECG)

L'ECG à 12 canaux sert à la détection de troubles de rythmes significatifs, surtout des fibrillations auriculaires [15] et de la maladie de l'oreillette avec bradycardies et tachyarythmies [16]. Toutes les deux sont liées à un risque thrombo-embolique accru. Le risque thrombo-embolique du flutter auriculaire est actuellement controversé [17]. Un ECG-holter de 24 heures est recommandé si on veut exclure une fibrillation auriculaire intermittente, mais uniquement en cas de suspicion clinique élevée ou en présence d'un ECG de routine non conclusif. Par ailleurs, l'ECG permet de poser le diagnostic d'infarctus subaigu ou chronique associé éventuellement à un anévrisme ventriculaire gauche qui pourrait être également responsable d'une complication thrombo-embolique.
L'échocardiographie (figure 7)

L'échocardiographie donne la possibilité d'évaluer l'anatomie, la fonction et l'hémodynamie cardiaque. L'échocardiographie trans-oesophagienne (ETO) et trans-thoracique (ETT) sont deux examens complémentaires pour l'évaluation des sources cardiaques emboligènes. L'échocardiographie trans-thoracique permet une bonne évaluation du ventricule gauche et de la fonction des prothèses valvulaires, par contre, l'ETT multiplanéaire permet de visualiser l'appareil valvulaire, les oreillettes et l'auricule gauche, le septum inter-atrial et l'aorte thoracique avec une résolution beaucoup plus précise. L'exclusion de source cardiaque emboligène est l'indication la plus fréquente pour procéder à une ETO chez 35 à 55\% de tous les patients [18]. 
Du point de vue échocardiographique, des sources cardiaques emboligènes peuvent être subdivisées en trois catégories [19]:

- Des structures qui portent un risque emboligène (des thrombi, des tumeurs, des plaques d'athérosclérose sur l'aorte thoracique, des végétations valvulaires).

- Des déficiences structurelles du septum interatrial avec prédisposition pour des embolies paradoxales (foramen ovale perméable, "patent foramen ovale est égal PFO».

- Des déficiences du septum interatrial, «atrial septal defect est égal à AFD».

- Prédisposition pour la formation d'un thrombus intra-cardiaque (contraste d'écho spontané, calcification de l'anneau de la valve mitrale, anévrisme du septum interatrial).

\section{Vice cardiaque structurel potentiellement} emboligène

Les thrombi originaires $d u$ ventricule gauche se forment le plus fréquemment dans des zones de myocarde dyskinésique consécutivement à un infarctus du myocarde ou en cas d'une fonction ventriculaire gauche diminuée dans le cadre d'une cardiomyopathie dilatée. Les thrombi du ventricule gauche peuvent

\section{Figure 4}

Angiographie de l'artère carotide gauche de la même patiente qui a été illustrée dans la figure 3. Il existe une artériosclérose sténosante de la carotide débutant à la bifurcation et qui s'étend à la fois au niveau de l'artère carotide interne et à la carotide externe. La paroi vasculaire est délimitée de façon irrégulière et elle est ulcérée. L'artère carotide est sténosée (sténose de 72\% selon la méthodeCC), (source: Unité de Neuroradiologie, Hôpital de l'île, Berne).

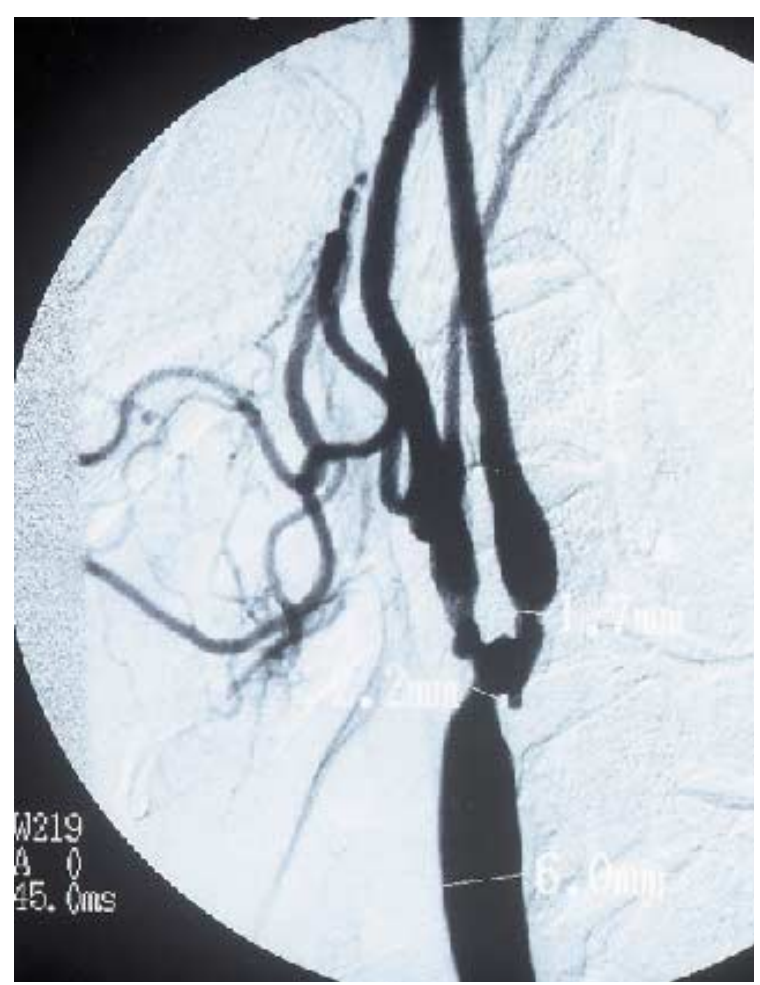

\section{Figure 5}

A Angiographie sélective d'un patient avec une hémiparésie aiguë gauche à prédominance facio-brachiale. Dans la région centrale certaines branches de l'artère cérébrale ne sont pas visualisées. Ceci indique leur occlusion.

B Après thrombolyse locale réussie, les branches précédemment qui ont été occlues de l'artère cérébrale moyenne réapparaissent à l'angiogramme, à comparer avec la figure 2 (source: Unité de Neuroradiologie, Hôpital de l'île, Berne).
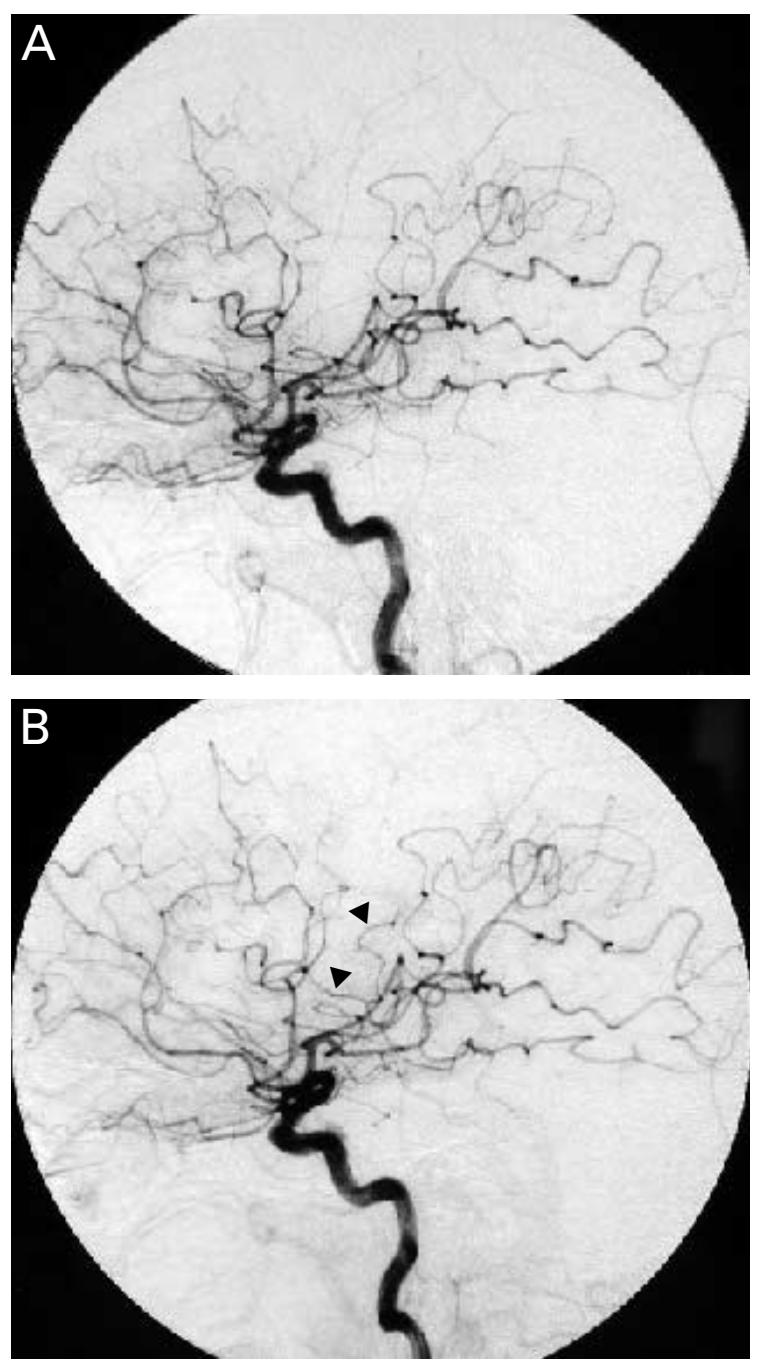

être démontrées à l'aide de l'ETT avec une sensibilité entre 75 et 95\%. Leur taille ainsi que leur mobilité sont associées à un risque emboligène accru. Si le résultat de l'ETT n'est pas concluant, l'ETO servira comme examen complémentaire. Dix pourcent des patients qui ont un thrombus dans le ventricule gauche démontré à l'échocardiographie, ont souffert d'une embolie cérébrale.

Des thrombi dans l'oreillette gauche constituent la source cardiaque emboligène la plus fréquente et sont le plus souvent liées à une fibrillation auriculaire, à une sténose mitrale d'origine rhumatismale ou encore à une cardiomyopathie dilatée. Alors que l'ETT offre seulement peu de sensibilité (40 à $60 \%$ pour la détection de thrombi de l'atrium), l'ET0 multiplanéaire est la méthode de choix pour l'évaluation de l'atrium 


\section{Figure 6}

A Angiographie cérébrale de l'artère carotide interne droite d'un patient de 57 avec un hémisyndrome sensitivo-moteur gauche plégique et une hémianopsie. Le tronc principal de l'artère cérébrale est occlue (voir flèche) et seulement l'artère cérébrale antérieure est visualisée.

B Après une thrombolyse réussie, le tronc principal de l'artère cérébrale moyenne et de la branche réapparaissent complètement. La patiente a complètement récupéré de son déficit neurologique et a repris son travail (source: Unité de Neuroradiologie, Hôpital de l'île, Berne).
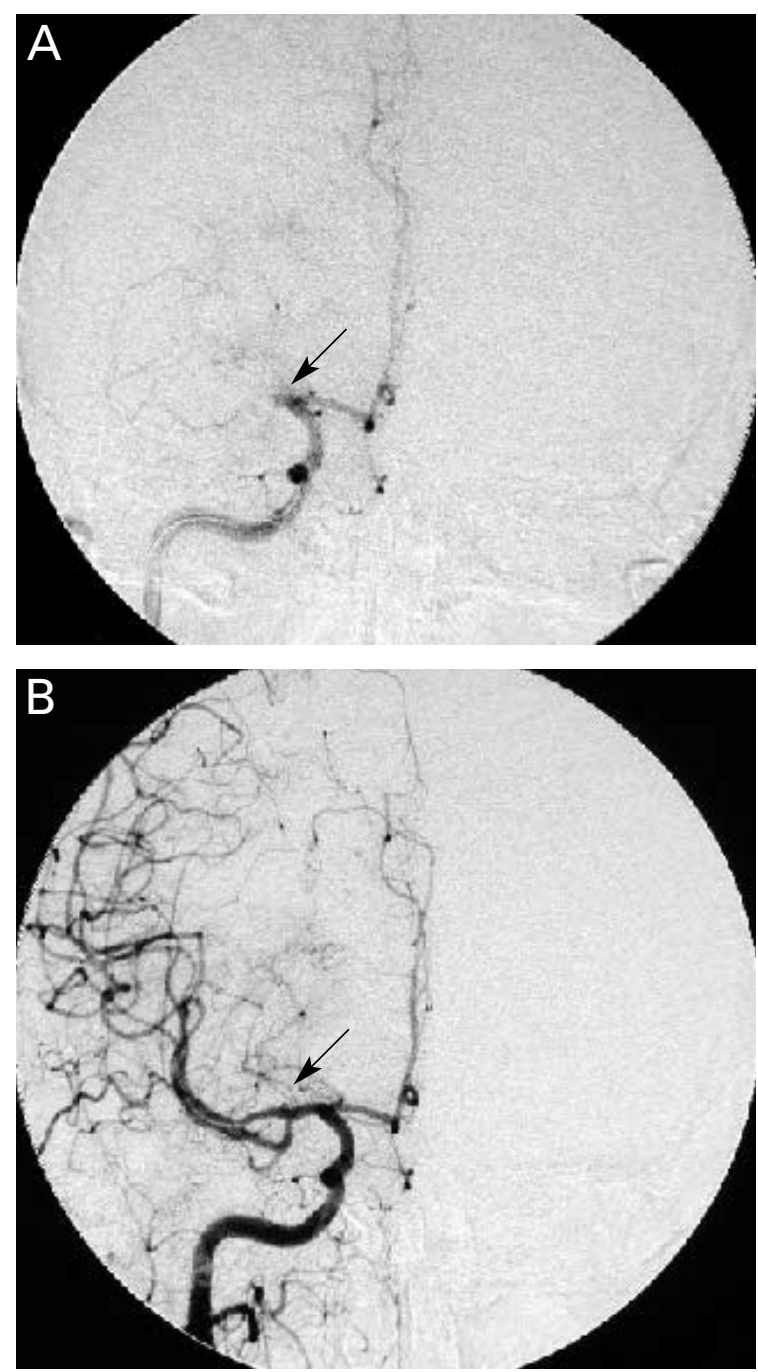

et de l'auricule gauche avec une sensibilité et une spécificité de l'ordre de plus de 95\% [20].

Des prothèses valvulaires mécaniques ou biologiques en position aortique ou mitrale peuvent être un foyer pour la formation de thrombi en cas d'anticoagulation insuffisante, et sont responsables d'embolies systémiques avec une incidence annuelle de 1 à $4 \%$ [21]. La démonstration de thrombi sur des prothèses valvulaires nécessitent dans la plupart des cas un ETO.

Des végétations valvulaires sont l'expression d'endocardites infectieuses ou non infectieuses (maladies inflammatoires chroniques, SIDA, cancers) et peuvent être la raison d'embolies cérébrales. L'ETO est la mé- thode de choix pour la démonstration de végétations sur des valves natives ou des prothèses valvulaires.

La première manifestation des tumeurs intracardiaques, qui sont dans l'ensemble rares, est un événement thrombo-embolique. Les myxomes constituent plus de 50\% de toutes les tumeurs intracardiaques et sont fixés le plus souvent dans la région de la fossa ovalis de la paroi gauche du septum interatrial [22]. De nombreuses tumeurs intracardiaques sont déjà identifiables à l'ETT, cependant l'ETO a une plus grande sensibilité et une meilleure définition concernant des détails structurels comme la taille, le contenu kystique, l'invasion ou la compression des structures avoisinantes. La sonde ETO permet une meilleure visualisation de la veine cave supérieure et inférieure et elle est ainsi supérieure concernant l'évaluation des thrombi localisés dans les voies veineuses centrales ou qui siègent sur les électrodes d'un pacemaker, potentiellement source d'embolies paradoxales en présence d'un foramen ovale perméable.

Des athéromes complexes de l'arc aortique et de l'aorte ascendante avec une épaisseur de l'intima de plus de $4 \mathrm{~mm}$ ont été associés à un risque accru d'AVC [23]. Des plaques aortiques mobiles présentent un risque emboligène élevé [24]. L'ETO multiplanéaire est la méthode de choix pour l'évaluation de l'aorte ascendante et de l'arc aortique.

\section{Déficience de la paroi du septum interatrial}

L'ETO permet une excellente visualisation de la paroi interatriale et ainsi des mécanismes emboligènes cardiaques associés. Un foramen ovale perméable ainsi qu'une défectuosité du septum interatrial, même de petite taille, permet un shunt temporaire droitegauche avec passage de thrombi veineux dans la circulation artérielle (embolies paradoxales) [25, 26], quand la pression de l'atrium droit est plus grande que celle de l'atrium gauche. Ce mécanisme physiopathologique peut être démontré grâce à l'échocardiographie de contraste par injection d'un mélange entre un liquide et des bulles d'air dans la veine cubitale, combinée à une manœuvre de Valsalva. L'anévrisme du septum interatrial est formé par un surplus de tissu au niveau de la fossa ovalis et entraîne une excavation sacciforme de la paroi septale dans l'oreillette droite ou gauche. La mobilité et l'amplitude maximales de la déviation de l'anévrisme du septum interatrial se documentent avec précision à l'ETO. Comme l'anévrisme de la paroi du septum interatrial est dans la plupart des cas associés à un foramen ovale perméable ou à une petite défectuosité du septum, le mécanisme physiopathologique le plus probable est également l'embolie paradoxale dans ce cas [27]. La théorie selon laquelle la paroi interatriale de l'anévrisme septal créerait des plis et des niches propices à la formation des thrombi, devrait être abandonnée.

\section{Prédisposition à la formation de thrombi intra- cardiaques}

La formation d'un contraste échogène spontané dans l'oreillette gauche ("smoke») est l'expression d'un flux sanguin ralenti dans le cadre d'une fibrillation auri- 


\section{Figure 7}

Echocardiographie trans-oesophagienne. Visualisation d'une grande lésion, écho-dense, occupant l'espace dans l'oreillette gauche, source d'embolie (myxome). OG est = oreillette gauche, VG est = ventricule gauche, VA est = valve aortique .

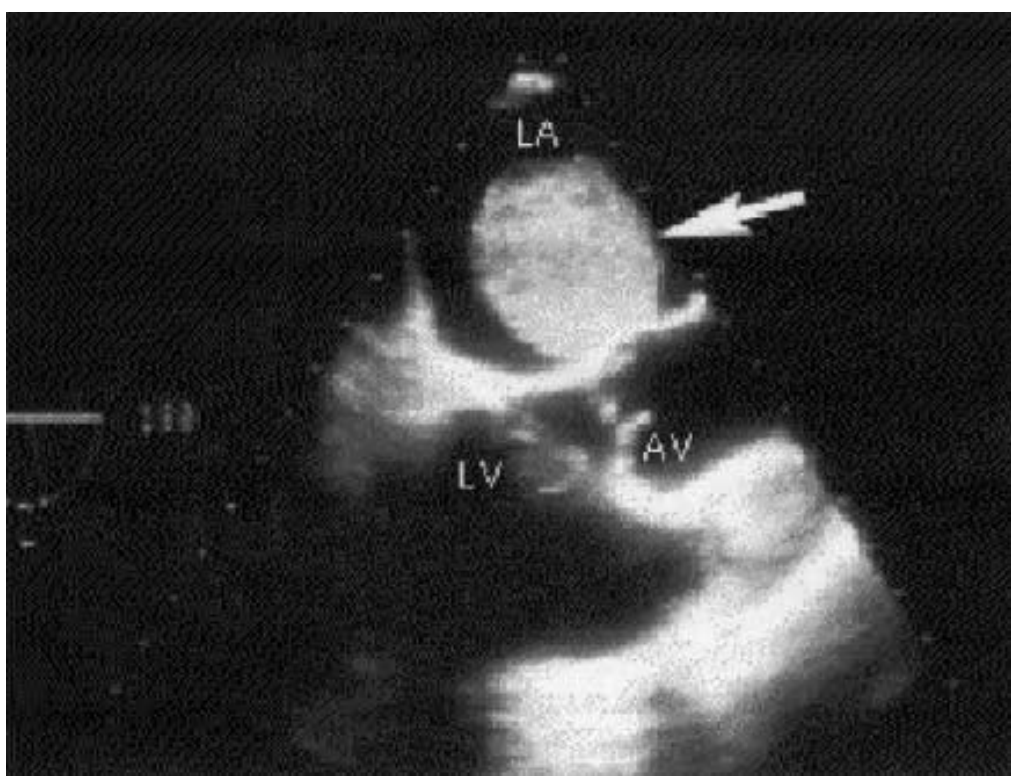

culaire, d'une sténose mitrale, d'une prothèse valvulaire en position mitrale ou d'une fonction ventriculaire gauche diminuée. La présence de contraste échogène spontané dans l'oreillette gauche est associée à un risque thrombo-embolique accru. Le mécanisme emboligène suspecté se base sur l'association avec des thrombi dans l'oreillette [28]. Le contraste échogène spontané peut être démontré de façon plus sensible à l'aide de l'ETO à cause de la fréquence d'émission élevée de la sonde d'ultrasons trans-oesophagienne par rapport à la sonde trans-thoracique.

Une calcification de l'anneau de la valve mitrale est associée à un risque élevé d'AVC [29]. Cependant, il n'est pas clair si l'anneau mitral calcifié est luimême à l'origine d'une formation de thrombi ou d'embolisation de particules calcaires ou s'il est seulement un marqueur pour un risque thrombo-embolique élevé de façon générale.

\section{Références}

1 Daniel WG, Mügge A. Transesophageal echocardiography. New Engl J Med 1995;332:1268-79.

2 Edelman RR, Warach S. Magnetic resonance imaging. N Engl J Med 1993;328:708-16 und 785-91.

3 Culebras A, Kase CS, Masdeu JC, Fox AJ, Bryan RN, Grossman CB, et al. Practice guidelines for the use of imaging in trasient ischemic attacks and acute stroke. A report of the Stroke Council, American Heart Association. Stroke 1996;28:1480-97.

4 Forsting M, Dörfler A, Knauth M, von Kummer R. Neuroradiologische Untersuchungen und Befunde beim Hirnschlag. Ther Umschau 1996;53:535-43.

5 Schellinger PD, Jansen 0, Fiebach JB, Hacke W, Sartor K. Standardized MRI stroke protocol. Comparison with CT in hyperacute intracerebral hemorrhage. Stroke 1999;30:765-8.
6 Von Kummer R, Holle R, Gzryska U, Hofmann E, Jansen 0, Petersen D, et al. Interobserver agreement in assessing $\mathrm{CT}$ signs of middle cerebral artery infarction. AJNR 1996; 17:1743-8.

7 Lövblad K0, Laubach HJ, Baird AE, Curtin F, Schlaug G, Edelman RR, et al. Clinical experience with diffusion-weighted MR in patients with acute stroke. AJNR 1998;19:1061-6.

8 Weber J, Mattle HP, Heid 0, Remonda L, Schroth G. Diffusion-weighted imaging in ischaemic stroke: a follow-up study. Neuroradiology 2000;42:184-191.

9 Patel MR, Edelman RR, Warach S. Detection of hyperacute primary intraparenchymal hemorrhage by magnetic resonance imaging. Stroke 1996;30:2321-4.

10 Von Büdingen HJ, von Reutern G-M. Ultraschaldiagnostik der hirnversorgenden Arterien. Stuttgart: Thieme; 1993.

11 Baumgartner RW, HP Mattle, R. Aaslid und M. Kaps. Transcranial color-coded duplex sonography in arterial cerebrovascular disease. Cerebrovasc Dis 1997;7:57-63.

12 Gönner F, Remonda L, Mattle HP, Sturzenegger M, Ozdoba C, Lövblad K0, et al. Local intra-arterial thrombolysis in acute schemic stroke. Stroke 1998;29:1894-900.

13 Cardiogenic brain embolism. The second report of the Cerebral Embolism Task Force. Arch Neurol 1989;46:727-43.

14 Brickner ME. Cardioembolic stroke. Am J Med 1996; 100:465-74.

15 Cardiogenic brain embolism. Cerebral Embolism Task Force. Arch Neurol 1986;43:71-84.

16 Fairfax AJ, Lambert CD, Leatham A. Systemic embolism in chronic sinoatrial disorder. N Engl J Med 1976;295:190-2.

17 Windecker S, Kay GN, Epstein AE, Plumb VJ. Atrial flutter. Cardiac Electrophysiology Review 1997;1:52-60.

18 Daniel WG, Mugge A. Transesophageal echocardiography. N Engl J Med 1995;332:1268-79.

19 Manning WJ. Role of transesophageal echocardiography in the management of thromboembolic stroke. Am J Cardiol 1997;80:19D-28D; discussion 35D-39D.

20 Manning WJ, Weintraub RM, Waksmonski CA, Haering JM, Rooney PS, Maslow AD, et al. Accuracy of transesophageal echocardiography for identifying left atrial thrombi.

A prospective, intraoperative study. Ann Intern Med 1995; 123:817-22.

21 Vongpatanasin W, Hillis LD, Lange RA. Prosthetic heart valves. N Engl J Med 1996;335:407-16.

22 Reynen K. Cardiac myxomas. N Engl J Med 1995;333:1610-7.

23 Amarenco P, Cohen A, Tzourio C, Bertrand B, Hommel M, Besson G, et al. Atherosclerotic disease of the aortic arch and the risk of ischemic stroke. N Engl J Med 1994;331:1474-9.

24 Karalis DG, Chandrasekaran K, Victor MF, Ross JJ, Jr., Mintz GS. Recognition and embolic potential of intraaortic atherosclerotic debris. J Am Coll Cardiol 1991;17:73-8.

25 Webster MW, Chancellor AM, Smith HJ, Swift DL, Sharpe DN, Bass NM, et al. Patent foramen ovale in young stroke patients. Lancet 1988;2:11-2.

26 Lechat P, Mas JL, Lascault G, Loron P, Theard M, Klimczac M, et al. Prevalence of patent foramen ovale in patients with stroke. N Engl J Med 1988;318:1148-52.

27 Agmon Y, Khandheria BK, Meissner I, Gentile F, Whisnant JP, Sicks JD, et al. Frequency of atrial septal aneurysms in patients with cerebral ischemic events. Circulation 1999;99:1942-4.

28 Daniel WG, Nellessen U, Schroder E, Nonnast-Daniel B, Bednarski P, Nikutta P, et al. Left atrial spontaneous echo contrast in mitral valve disease: an indicator for an increased thromboembolic risk. J Am Coll Cardiol 1988;11:1204-11.

29 Benjamin EJ, Plehn JF, D’Agostino RB, Belanger AJ, Comai K Fuller DL, et al. Mitral annular calcification and the risk of stroke in an elderly cohort. N Engl J Med 1992;327:374-9.

Des tirés à part peuvent être obtenus dès automne 2000 auprès la Fondation suisse de cardiologie, Schwarztorstrasse 14, Case postale, 3000 Berne 14.

Deutsch erschienen in Nr. 14/2000 